\title{
APPROXIMATE CONTROLLABILITY OF DELAYED SEMILINEAR CONTROL SYSTEMS
}

\author{
LIANWEN WANG
}

Received 22 January 2004 and in revised form 9 July 2004

We deal with the approximate controllability of control systems governed by delayed semilinear differential equations $\dot{y}(t)=A y(t)+A_{1} y(t-\Delta)+F\left(t, y(t), y_{t}\right)+(B u)(t)$. Various sufficient conditions for approximate controllability have been obtained; these results usually require some complicated and limited assumptions. Results in this paper provide sufficient conditions for the approximate controllability of a class of delayed semilinear control systems under natural assumptions.

\section{Introduction}

The main concern in this paper is the approximate controllability of the following delayed semilinear control system:

$$
\begin{gathered}
\dot{y}(t)=A y(t)+A_{1} y(t-\Delta)+F\left(t, y(t), y_{t}\right)+(B u)(t), \quad t \geq a, \\
y_{a}=\xi,
\end{gathered}
$$

in a real Hilbert space $X$ with the norm $\|\cdot\|$. The meaning of all notations is listed as follows: $\Delta \geq 0$ is a system delay; $y(\cdot):[a-\Delta, b] \rightarrow X$ is the state function; $\xi \in C([-\Delta, 0] ; X)$, the Banach space of all continuous functions $\psi:[-\Delta, 0] \rightarrow X$ endowed with the norm $|\psi|=\sup \{\|\psi(\theta)\|:-\Delta \leq \theta \leq 0\} ; A$ is the generator of a $C_{0}$ semigroup $T(t)$ in $\mathrm{X} ; A_{1}$ is a bounded linear operator from $X$ to $X ; F:[a, b] \times X \times C([-\Delta, 0] ; X) \rightarrow X$ is a nonlinear operator; $u(\cdot) \in L^{2}(a, b ; U)$ is a control function; $U$ is a Hilbert space; $B$ is a bounded linear operator from $L^{2}(a, b ; U)$ to $L^{2}(a, b ; X)$. In addition, for any $y \in C([a-\Delta, b] ; X)$ and $t \in[a, b]$, define $y_{t} \in C([-\Delta, 0] ; X)$ by $y_{t}(\theta)=y(t+\theta)$ for $\theta \in[-\Delta, 0]$.

Denote the state function of $(1.1)$ corresponding to a control $u(\cdot)$ by $y(\cdot ; u)$. Then $y(b ; u)$ is the state value at terminal time $b$. Introduce the set

$$
R_{b}(F)=\left\{y(b ; u): u(\cdot) \in L^{2}(a, b ; U)\right\}
$$

which is called the reachable set of system (1.1) at terminal time $b$, its closure in $X$ is denoted $\overline{R_{b}(F)}$. 
Definition 1.1. The system (1.1) is said to be approximately controllable on $[a, b]$ if $\overline{R_{b}(F)}=X$.

The following system is called the corresponding linear system of (1.1):

$$
\begin{gathered}
\dot{y}(t)=A y(t)+A_{1} y(t-\Delta)+(B u)(t), \quad t \geq a, \\
y_{a}=\xi .
\end{gathered}
$$

This is a special case of (1.1) with $F \equiv 0$. The reachable set of system (1.3) at terminal time $b$ is denoted $R_{b}(0)$. Similarly, system $(1.3)$ is said to be approximately controllable on $[a, b]$ if $\overline{R_{b}(0)}=X$.

For semilinear control systems without delays, approximate controllability has been extensively studied in the literature. We list only a few of them. Zhou [10] studied the approximate controllability for a class of semilinear abstract equations. Naito [6] established the approximate controllability for semilinear control systems under the assumption that the nonlinear term is bounded. Approximate controllability for semilinear control systems also can be found in Choi et al. [1], Fernandez and Zuazua [2], Li and Yong [4], Mahmudov [5], and many other papers. Most of them concentrate on finding conditions of $F, A$, and $B$ such that semilinear systems are approximately controllable on $[a, b]$ if the corresponding linear systems are approximately controllable on $[a, b]$.

For semilinear delayed control systems, some papers are devoted to the approximate controllability. For example, Klamka [3] provided some approximate controllability results. Naito and Park [7] dealt with approximate controllability for delayed Volterra systems. In [9] Ryu et al. studied approximate controllability for delayed Volterra control systems. The purpose of this paper is to study the approximate controllability of control system (1.1). We obtain the approximate controllability of system (1.1) if the corresponding linear system is approximate controllable and other natural assumptions such as the local Lipschitz continuity for $F$ and the compactness of operator $W$ are satisfied.

\section{Basic assumptions}

We start this section by introducing the fundamental solution $S(t)$ of the following system:

$$
\begin{gathered}
\dot{y}(t)=A y(t)+A_{1} y(t-\Delta), \quad t \geq a, \\
y_{a}=\xi .
\end{gathered}
$$

We already know that (2.1) has a unique solution, denoted by $y^{\xi}(t)$, for each $\xi \in C([-\Delta$, $0] ; X)$. Hence, we can define an operator $S(t)$ in $X$ by

$$
S(t) \xi(0)= \begin{cases}y^{\xi}(t+a), & t \geq 0 \\ 0, & t<0\end{cases}
$$


$S(t)$ is called the fundamental solution of (2.1). It is easy to check that $S(t)$ is the unique solution of the following operator equation:

$$
S(t)=T(t)+\int_{0}^{t} T(t-s) A_{1} S(s-\Delta) d s .
$$

Let $K:=\max \{\|T(t)\|: 0 \leq t \leq b\}$. By (2.3) we have

$$
\|S(t)\| \leq K+\int_{0}^{t} K\left\|A_{1}\right\|\|S(s-\Delta)\| d s \leq K+K\left\|A_{1}\right\| \int_{\Delta}^{t}\|S(s)\| d s .
$$

Gronwall's inequality implies that

$$
\|S(t)\| \leq K \exp (K\|A\|(b-\Delta)):=M_{1}, \quad 0 \leq t \leq b .
$$

Throughout the paper we impose the following condition on $F$.

(H1) $F:[a, b] \times X \times C([-\Delta, 0] ; X) \rightarrow X$ is locally Lipschitz continuous in $y, \eta$ uniformly in $t \in[a, b]$; that is, for any $r>0$, there is a constant $L(r)$ such that

$$
\left\|F\left(t, y_{1}, \eta_{1}\right)-F\left(t, y_{2}, \eta_{2}\right)\right\| \leq L(r)\left(\| y_{1}-y_{2}||+\left|\eta_{1}-\eta_{2}\right|\right)
$$

for any $t \in[a, b],\left\|y_{1}\right\| \leq r,\left\|y_{2}\right\| \leq r,\left|\eta_{1}\right| \leq r$, and $\left|\eta_{2}\right| \leq r$.

With a minor modification of $[8]$, we can prove that system (1.1) has a unique mild solution $y(\cdot ; u) \in C([a-\Delta, b] ; X)$ for any control $u(\cdot) \in L^{2}(a, b ; U)$ under assumption (H1). This mild solution is defined as a solution of the integral equation:

$$
\begin{gathered}
y(t ; u)=S(t-a) \xi(0)+\int_{a}^{t} S(t-s)\left[F\left(s, y(s ; u), y_{s}\right)+(B u)(s)\right] d s, \quad t \geq a, \\
y_{a}=\xi .
\end{gathered}
$$

Similarly, for any $z(\cdot) \in L^{2}(a, b ; X)$, the following integral equation:

$$
\begin{gathered}
x(t ; z)=S(t-a) \xi(0)+\int_{a}^{t} S(t-s)\left[F\left(s, x(s ; z), x_{s}\right)+z(s)\right] d s, \quad t \geq a, \\
x_{a}=\xi
\end{gathered}
$$

has a unique mild solution $x(\cdot ; z)$. Therefore, we can define an operator $W$ from $L^{2}(a, b ; X)$ to $C([a, b] ; X)$ by

$$
(W z)(\cdot)=x(\cdot ; z)
$$

Regarding the operator $W$, we assume that

(H2) $W$ is a compact operator.

Remark 2.1. (H2) is the case if, for instance, $T(t)$, the semigroup generated by $A$, is a compact semigroup.

The following assumption (H3) was introduced by Naito in [6]. Define a linear operator $\varphi$ from $L^{2}(a, b ; X)$ to $X$ by

$$
\varphi p=\int_{a}^{b} S(b-s) p(s) d s \quad \text { for } p(\cdot) \in L^{2}(a, b ; X) .
$$


Let the kernel of the operator $\varphi$ be $N$; that is, $N=\{p: \varphi p=0\}$. Then $N$ is a closed subspace of $L^{2}(a, b ; X)$. Denote its orthogonal space in $L^{2}(a, b ; X)$ by $N^{\perp}$. Let $G$ be the projection operator from $L^{2}(a, b ; X)$ into $N^{\perp}$ and let $R[B]$ be the range of $B$. We assume that

(H3) for any $p(\cdot) \in L^{2}(a, b ; X)$, there is a function $q(\cdot) \in \overline{R[B]}$ such that $\varphi p=\varphi q$.

Remark 2.2. (H3) is valid for many control systems, see [6] for detailed discussion.

It follows from assumption (H3) that $\{x+N\} \cap \overline{R[B]} \neq \varnothing$ for any $x \in N^{\perp}$. Therefore, the operator $P$ from $N^{\perp}$ to $\overline{R[B]}$ defined by

$$
P x=x^{*},
$$

where $x^{*} \in\{x+N\} \cap \overline{R[B]}$ and $\left\|x^{*}\right\|=\min \{\|y\|: y \in\{x+N\} \cap \overline{R[B]}\}$, is well defined. It is proved in [6] that $P$ is bounded.

\section{Lemmas}

This section provides two lemmas that will be used to prove the main theorem.

Lemma 3.1. Assume that $a(t)$ is continuous on $[a, b], b(t)$ is nonnegative and integrable on $[a, b]$, and $x(t)$ is a nonnegative continuous function satisfying the following inequality:

$$
x(t) \leq a(t)+\int_{a}^{t} b(s) x^{\alpha}(s) d s, \quad 0 \leq \alpha<1, t \in[a, b] .
$$

If the equation

$$
y(t)=a(t)+\int_{a}^{t} b(s) y^{\alpha}(s) d s
$$

has a unique solution $\bar{y}(t)$ on $[a, b]$, then

$$
x(t) \leq \bar{y}(t), \quad t \in[a, b] .
$$

Proof. Let $C[a, b]$ be the Banach space of all continuous functions on $[a, b]$ endowed with the maximum norm. Define an operator $E$ from $C[a, b]$ to $C[a, b]$ by

$$
(E y)(t)=a(t)+\int_{a}^{t} b(s) y^{\alpha}(s) d s .
$$

Construct a sequence $\left\{y_{n}\right\}$ as follows:

$$
y_{0}(t)=x(t), \quad y_{n+1}(t)=\left(E y_{n}\right)(t), \quad n=0,1, \ldots
$$

We have

$$
x(t)=y_{0}(t) \leq y_{1}(t) \leq \cdots, \quad\|x\|=\left\|y_{0}\right\| \leq\left\|y_{1}\right\| \leq \cdots .
$$

Note that

$$
\|E y\| \leq\|a\|+\|y\|^{\alpha} \int_{0}^{t} b(s) d s
$$


then we can find a number $d>0$ such that

$$
\|E y\| \leq\|y\| \quad \text { for }\|y\| \geq d
$$

If $\left\|y_{n}\right\| \leq d$ holds for any integer $n=0,1, \ldots$, then $\left\|y_{n}\right\|$ is bounded. Otherwise, it follows from (3.6) that a sufficiently large integer $N$ exists such that

$$
\left\|y_{0}\right\| \leq \cdots \leq\left\|y_{N}\right\| \leq d, \quad\left\|y_{n}\right\|>d \quad \text { for } n>N \text {. }
$$

Thus

$$
\max \left\{\left\|y_{n}\right\|: n \geq 0\right\} \leq \max \left\{d,\left\|y_{N+1}\right\|\right\}:=m
$$

Consequently,

$$
0 \leq x(t)=y_{0}(t) \leq y_{1}(t) \leq \cdots \leq y_{n}(t) \leq \cdots \leq m
$$

Therefore,

$$
\lim _{n \rightarrow \infty} y_{n}(y)=\bar{y}(t)
$$

Note that $\bar{y}(t)$ is the unique solution of (3.2). The conclusion of Lemma 3.1 follows from (3.11).

Lemma 3.2. Assume that (H1) is fulfilled. Furthermore, for any $y \in X$ and $\eta \in C([-\Delta, 0] ; X)$

$$
\|F(t, y, \eta)\| \leq M\left(1+\|y\|^{\alpha}+|\eta|^{\alpha}\right), \quad 0 \leq \alpha<1, t \in[a, b] .
$$

Then the mild solution $x(t ; z)$ of $(2.8)$ has the estimate

$$
\left|x_{t}\right| \leq H(\|z\|)
$$

where $H(r)$ is an increasing function and $H(r)=O(r)$ as $r \rightarrow \infty$.

Proof. Recall that

$$
M_{1}=\max \{\|S(t)\|: 0 \leq t \leq b\} .
$$

It follows from $\|x(s)\| \leq\left|x_{s}\right|$ and (2.8) that

$$
\begin{aligned}
\|x(t)\| & \leq M_{1}|\xi|+M_{1} M \int_{a}^{t}\left(1+\|x(s)\|^{\alpha}+\left|x_{s}\right|^{\alpha}\right) d s+M_{1} \int_{a}^{t}\|z(s)\| d s \\
& \leq M_{1}|\xi|+M_{1} M(b-a)+M_{1} \sqrt{b-a}\|z\|+2 M_{1} M \int_{a}^{t}\left|x_{s}\right|^{\alpha} d s .
\end{aligned}
$$

For any $\theta \in[-\Delta, 0]$, we have

$$
\|x(t+\theta)\| \leq M_{1}|\xi|+M_{1} M(b-a)+M_{1} \sqrt{b-a}\|z\|+2 M_{1} M \int_{a}^{t+\theta}\left|x_{s}\right|^{\alpha} d s .
$$


72 Approximate controllability of delayed systems

Hence

$$
\left|x_{t}\right| \leq M_{1}|\xi|+M_{1} M(b-a)+M_{1} \sqrt{b-a}\|z\|+2 M_{1} M \int_{a}^{t}\left|x_{s}\right|^{\alpha} d s .
$$

Note that for any two constants $V_{1}$ and $V_{2}$, the following equation

$$
y(t)=V_{1}+V_{2} \int_{0}^{t} y^{\alpha}(s) d s
$$

has a unique solution

$$
y(t)=\left[(1-\alpha) V_{2} t+V_{1}^{1-\alpha}\right]^{1 /(1-\alpha)} .
$$

Applying Lemma 3.1 to (3.18), we obtain

$$
\begin{aligned}
\left|x_{t}\right| & \leq\left[2(1-\alpha) M M_{1}(b-a)+\left(M_{1}|\xi|+M_{1} \sqrt{b-a}\|z\|+M M_{1}(b-a)\right)^{1-\alpha}\right]^{1 /(1-\alpha)} \\
& :=H(\|z\|) .
\end{aligned}
$$

Clearly, the function $H(r)$ satisfies all requirements of Lemma 3.2 and the proof of the lemma is complete.

\section{Approximate controllability}

The following theorem is the main result of this paper.

Theorem 4.1. Assume that linear system (1.3) is approximately controllable on $[a, b]$. If (H1), (H2), (H3), and (3.13) are fulfilled, then system (1.1) is approximately controllable on $[a, b]$.

Proof. Note that system (1.3) is approximately controllable on $[a, b]$ by the assumption, then $\overline{R_{b}(0)}=X$. To prove the approximate controllability of $(1.1)$; that is, $\overline{R_{b}(F)}=X$, it is sufficient to show that

$$
R_{b}(0) \subset \overline{R_{b}(F)}
$$

That means for any $\epsilon>0$ and $x^{b} \in R_{b}(0)$, there exists a $\nu \in R_{b}(F)$ such that $\left\|\nu-x^{b}\right\|<\epsilon$.

By the definition of reachable set $R_{b}(0)$ of system $(1.3)$, there is a control $u(\cdot) \in$ $L^{2}(a, b ; U)$ such that

$$
x^{b}=S(b-a) \xi(0)+\int_{a}^{b} S(b-s)(B u)(s) d s .
$$

Let $\bar{z}_{0}=B u, z_{0}=G \bar{z}_{0}$. Then $z_{0} \in N^{\perp}$. Define an operator $J$ from $N^{\perp}$ to $N^{\perp}$ by

$$
J v=z_{0}-G \Gamma P v, \quad v \in N^{\perp}
$$


where $\Gamma$ is the operator from $L^{2}(a, b ; X)$ to $L^{2}(a, b ; X)$ defined by

$$
(\Gamma z)(t):=F\left(t,(W z)(t),(W z)_{t}\right)=F\left(t, x(t ; z), x_{t}\right) .
$$

For any $v \in N^{\perp}$, we have $P v \in L^{2}(a, b ; X), \Gamma P v \in L^{2}(a, b ; X)$, and $G \Gamma P v \in N^{\perp}$. Therefore, $J$ is well defined.

Since $W$ is compact by assumption (H2), for any bounded sequence $z_{n}(\cdot) \in L^{2}(a, b ; X)$; that is, $\left\|z_{n}\right\| \leq r_{1}$ for some $r_{1}>0$, there is a subsequence $z_{n_{k}}(\cdot)$ of $z_{n}(\cdot)$ such that $\left(W z_{n_{k}}\right)(\cdot)$ converges to $\bar{x}(\cdot)$ in $C([a, b] ; X)$ as $k \rightarrow \infty$. So, $W z_{n_{k}}$ is bounded in $C([a, b] ; X)$; that is, $\left\|W z_{n_{k}}\right\| \leq r_{2}$ for some constant $r_{2}>0$. (H1) implies that a constant $L(r)>0$ exists such that

$$
\begin{aligned}
& \left\|F\left(t,\left(W z_{n_{k}}\right)(t),\left(W z_{n_{k}}\right)_{t}\right)-F\left(t, \bar{x}(t), \bar{x}_{t}\right)\right\| \\
& \quad \leq L(r)\left(\left\|\left(W z_{n_{k}}\right)(t)-\bar{x}(t)\right\|+\left|\left(W z_{n_{k}}\right)_{t}-\bar{x}_{t}\right|\right),
\end{aligned}
$$

where $r=\max \left(r_{1}, r_{2}\right)$. Hence, we have

$$
\begin{aligned}
\left\|\Gamma z_{n_{k}}-F(\cdot, \bar{x}(\cdot), \bar{x} .)\right\|^{2} & \\
= & \left\|F\left(\cdot,\left(W z_{n_{k}}\right)(\cdot),\left(W z_{n_{k}}\right) .\right)-F(\cdot, \bar{x}(\cdot), \bar{x} .)\right\|^{2} \\
\leq L^{2}(r)(b-a)\left(\sup _{a \leq t \leq b}\left\|\left(W z_{n_{k}}\right)(t)-\bar{x}(t)\right\|\right. & \\
& \left.\quad \sup _{a \leq t \leq b}\left|\left(W z_{n_{k}}\right)_{t}-\bar{x}_{t}\right|\right)^{2} \longrightarrow 0
\end{aligned}
$$

as $k \rightarrow \infty$. Therefore, $\Gamma$ is compact and $J$ is compact as well.

From Lemma 3.2, for any $z(\cdot) \in L^{2}(a, b ; X)$, we have

$$
\left\|F\left(t, x(t ; z), x_{t}\right)\right\| \leq M\left(1+\|x(t ; z)\|^{\alpha}+\left|x_{t}\right|^{\alpha}\right) \leq M\left(1+2 H^{\alpha}(\|z\|)\right) .
$$

Note that $H(r)$ is increasing and $P$ is a bounded operator, then

$$
\begin{aligned}
\left\|z_{0}-G \Gamma P v\right\| & \leq\left\|z_{0}\right\|+\|G \Gamma P v\| \\
& \leq\left\|z_{0}\right\|+M \sqrt{b-a}+2 M \sqrt{b-a} H^{\alpha}(\|P\|\|v\|) .
\end{aligned}
$$

Taking into account

$$
\lim _{\|v\| \rightarrow \infty} \frac{\left\|z_{0}\right\|+M \sqrt{b-a}+2 M \sqrt{b-a} H^{\alpha}(\|P\|\|v\|)}{\|v\|}=0,
$$

then

$$
\lim _{\|v\| \rightarrow \infty} \frac{\left\|z_{0}-G \Gamma P v\right\|}{\|v\|}=0 .
$$

Therefore, we can find a sufficiently large number $\bar{r}$ such that

$$
\left\|z_{0}-G \Gamma P v\right\| \leq \bar{r} \quad \text { for }\|v\| \leq \bar{r} .
$$


This means that $J$ maps the bounded closed set $D(\bar{r})=\left\{v:\|v\| \leq \bar{r}, v \in N^{\perp}\right\}$ of $N^{\perp}$ into itself. Consequently, a fixed point of operator $J$ exists due to the Schauder fixed point theorem; that is, there is a $v^{*} \in D(\bar{r})$ such that

$$
J v^{*}=z_{0}-G \Gamma P v^{*}=v^{*}
$$

On account of

$$
P v^{*} \in\left(v^{*}+N\right) \cap \overline{R[B]},
$$

we have

$$
\int_{a}^{b} S(b-s)\left(P v^{*}\right)(s) d s=\int_{a}^{b} S(b-s) v^{*}(s) d s .
$$

Note that $G$ is the projection operator from $L^{2}(0, T ; X)$ into $N^{\perp}$, then we have

$$
\begin{aligned}
\int_{a}^{b} S(b-s) G p(s) d s & =\int_{a}^{b} S(b-s) p(s) d s \quad \text { for } p(\cdot) \in L^{2}(a, b ; X), \\
\int_{a}^{b} S(b-s)(B u)(s) d s & =\int_{a}^{b} S(b-s)\left[F\left(s, x\left(s ; P v^{*}\right), x_{s}\right)+v^{*}(s)\right] d s \\
& =\int_{a}^{b} S(b-s)\left[F\left(s, x\left(s ; P v^{*}\right), x_{s}\right)+\left(P v^{*}\right)(s)\right] d s .
\end{aligned}
$$

Finally,

$$
x^{b}=S(b-a) \xi(0)+\int_{a}^{b} S(b-s)\left[F\left(s, x\left(s ; P v^{*}\right), x_{s}\right)+\left(P v^{*}\right)(s)\right] d s=x\left(b ; P v^{*}\right) .
$$

Observe that $P v^{*} \in \overline{R[B]}$, then there is a sequence $u_{n}(\cdot) \in L^{2}(a, b ; U)$ such that $B u_{n} \rightarrow$ $P v^{*}$ as $n \rightarrow \infty$. W is continuous due to its compactness, then

$$
W B u_{n} \longrightarrow W P v^{*} \quad \text { in } C([a, b] ; X) \text {. }
$$

This implies

$$
x\left(b ; B u_{n}\right) \longrightarrow x\left(b ; P v^{*}\right)=x^{b}
$$

as $n \rightarrow \infty$. Since $x\left(b ; B u_{n}\right)=y\left(b ; u_{n}\right) \in R_{T}(F)$, we obtain $x^{b} \in \overline{R_{T}(F)}$ and complete the proof of the theorem.

Remark 4.2. If $A_{1}=0,(\mathrm{H} 3)$ implies the approximate controllability of $(1.3)$ on $[a, b]$ (see [6]). Therefore, Naito's result in [6] is a special case of Theorem 4.1 when $A_{1}=0, \Delta=$ 0 , and $F\left(t, x(t), x_{t}\right)=F(x(t))$. In particular, we improve Naito's result by weakening the uniform Lipschitz continuity and the uniform boundedness imposed on the nonlinear term. 


\section{Example}

Let $X=L^{2}(0, \pi)$ and $e_{n}(x)=\sin (n x)$ for $n=1, \ldots$ Then $\left\{e_{n}: n=1,2, \ldots\right\}$ is an orthogonal base for $X$. Define $A: X \rightarrow X$ by $A y=y^{\prime \prime}$ with domain

$$
D(A)=\left\{y \in X: y \text { and } y^{\prime} \text { are absolutely continuous, } y^{\prime \prime} \in X, y(0)=y(\pi)=0\right\} .
$$

Then

$$
A y=-\sum_{n=1}^{\infty} n^{2}\left\langle y, e_{n}\right\rangle e_{n}, \quad y \in D(A) .
$$

It is well known that $A$ is the infinitesimal generator of an analytic group $T(t), t \geq 0$, in $X$ and is given by

$$
T(t) y=\sum_{n=1}^{\infty} e^{-n^{2} t}\left\langle y, e_{n}\right\rangle e_{n}, \quad y \in X .
$$

$T(t)$ is compact because it is an analytic semigroup. Define an infinite dimensional space $U$ by

$$
U=\left\{u: u=\sum_{n=2}^{\infty} u_{n} e_{n}, \sum_{n=2}^{\infty} u_{n}^{2}<\infty\right\}
$$

with the norm defined by

$$
\|u\|_{U}=\left(\sum_{n=2}^{\infty} u_{n}^{2}\right)^{1 / 2}
$$

Define a mapping $B$ from $U$ to $X$ as follows:

$$
B u=2 u_{2} e_{1}+\sum_{n=2}^{\infty} u_{n} e_{n} .
$$

Consider the following delayed semilinear heat equation:

$$
\begin{aligned}
\frac{\partial y(t, x)}{\partial t}= & \frac{\partial^{2} y(t, x)}{\partial x^{2}}+y(t-\Delta, x)+F(y(t, x), y(t-\Delta, x)) \\
& +B u(t, x), \quad 0<t<b, 0<x<\pi, \\
y(t, 0)= & y(t, \pi)=0, \quad 0 \leq t \leq b, \\
y(t, x)= & \xi(x), \quad-\Delta \leq t \leq 0,0 \leq x \leq \pi .
\end{aligned}
$$

Then system (5.7) can be written to the abstract form (1.1). (H2) holds because $T(t)$ is a compact semigroup. Following the same arguments as in [6] we can prove that (H3) is valid and that the corresponding linear system is approximately controllable on $[0, b]$. By Theorem 4.1, system (5.7) is approximately controllable on $[0, b]$ if $F$ is locally Lipschitz continuous and condition (3.13) is satisfied. 


\section{Approximate controllability of delayed systems}

\section{References}

[1] J. R. Choi, Y. C. Kwun, and Y. K. Sung, Approximate controllability for nonlinear integrodifferential equations, J. Korea Soc. Math. Educ. Ser. B Pure Appl. Math. 2 (1995), no. 2, 173-181.

[2] L. A. Fernández and E. Zuazua, Approximate controllability for the semilinear heat equation involving gradient terms, J. Optim. Theory Appl. 101 (1999), no. 2, 307-328.

[3] J. Klamka, Controllability of Dynamical Systems, Mathematics and its Applications (East European Series), vol. 48, Kluwer Academic Publishers Group, Dordrecht, 1991.

[4] X. Li and J. Yong, Optimal Control Theory for Infinite Dimensional Systems, Birkhauser, Boston, Massachusettes, USA, 1995.

[5] N. I. Mahmudov, Approximate controllability of semilinear deterministic and stochastic evolution equations in abstract spaces, SIAM J. Control Optim. 42 (2003), no. 5, 1604-1622.

[6] K. Naito, Controllability of semilinear control systems dominated by the linear part, SIAM J. Control Optim. 25 (1987), no. 3, 715-722.

[7] K. Naito and J. Y. Park, Approximate controllability for trajectories of a delay Volterra control system, J. Optim. Theory Appl. 61 (1989), no. 2, 271-279.

[8] A. Pazy, Semigroups of Linear Operators and Applications to Partial Differential Equations, Applied Mathematical Sciences, vol. 44, Springer-Verlag, New York, 1983.

[9] J. W. Ryu, J. Y. Park, and Y. C. Kwun, Approximate controllability of delay Volterra control system, Bull. Korean Math. Soc. 30 (1993), no. 2, 277-284.

[10] H. X. Zhou, Approximate controllability for a class of semilinear abstract equations, SIAM J. Control Optim. 21 (1983), no. 4, 551-565.

Lianwen Wang: Department of Mathematics and Computer Science, Central Missouri State University, Warrensburg, MO 64093, USA

E-mail address: lwang@cmsul.cmsu.edu 


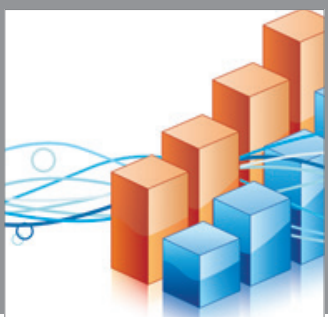

Advances in

Operations Research

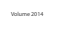

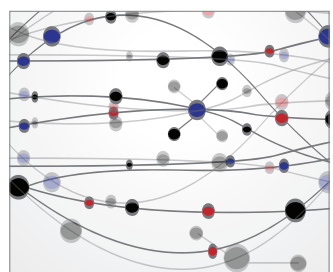

\section{The Scientific} World Journal
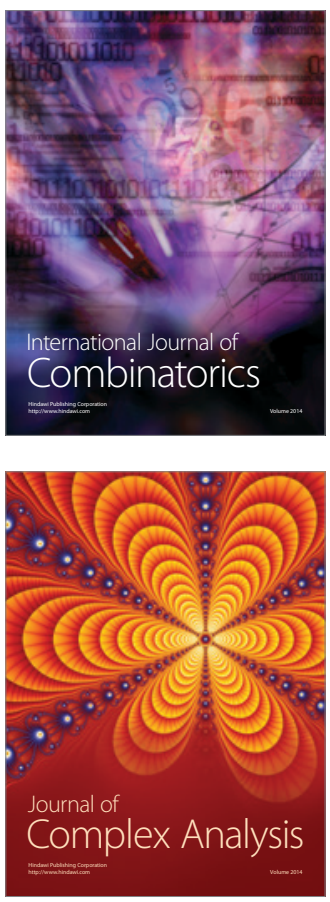

International Journal of

Mathematics and

Mathematical

Sciences
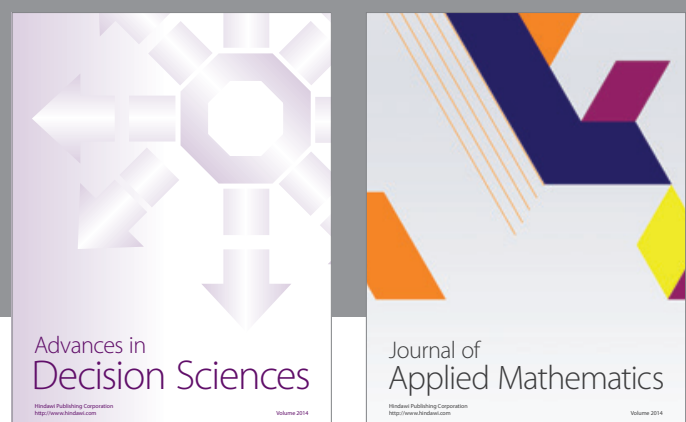

Journal of

Applied Mathematics
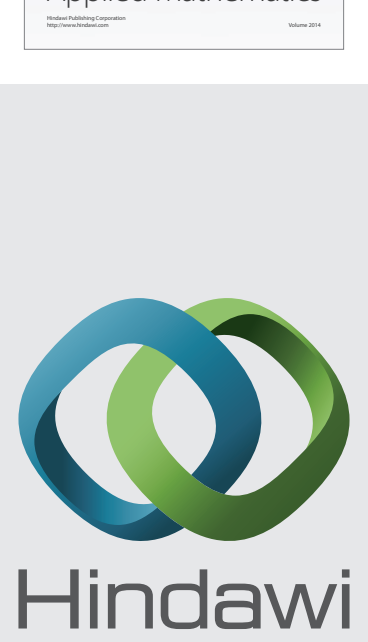

Submit your manuscripts at http://www.hindawi.com
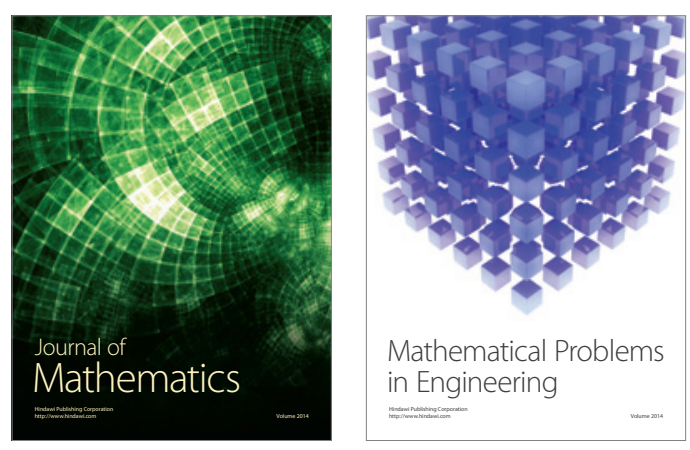

Mathematical Problems in Engineering
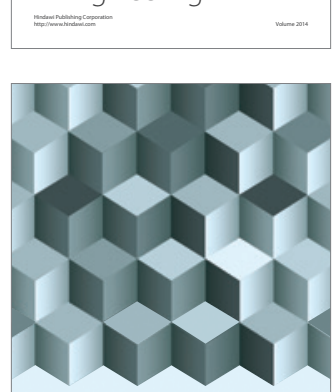

Journal of

Function Spaces
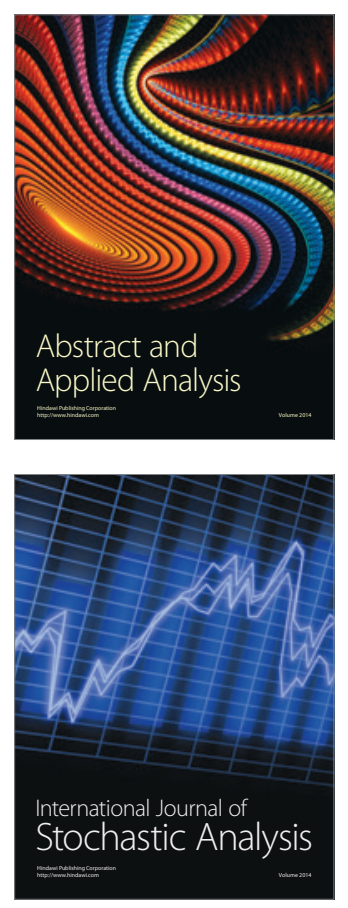

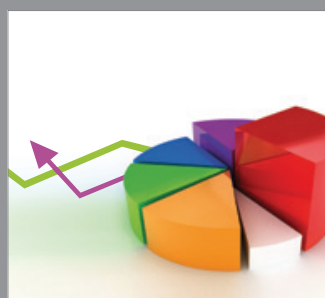

ournal of

Probability and Statistics

Promensencen
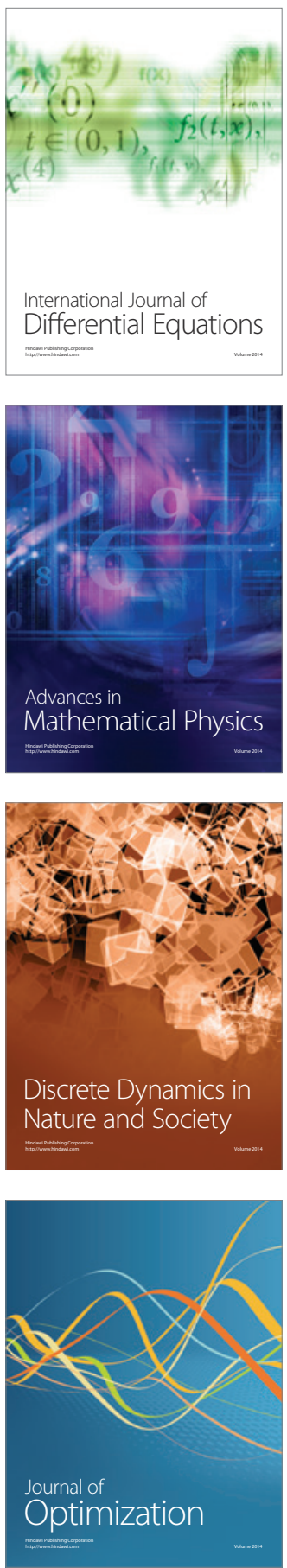